\title{
RANK OF SUBMATRICES OF THE PASCAL MATRIX
}

\section{SCOTT KERSEY}

Department of Mathematical Science

Georgia Southern University

Statesboro, GA 30460-8093

USA

e-mail: scott.kersey@gmail.com

\begin{abstract}
In a previous paper, we derived necessary and sufficient conditions for the invertibility of square submatrices of the Pascal upper triangular matrix. To do so, we established a connection with the two-point Birkhoff interpolation problem. In this paper, we extend this result by deriving a formula for the rank of submatrices of the Pascal matrix. Our formula works for both square and nonsquare submatrices. We also provide bases for the row and column spaces of these submatrices. Further, we apply our result to one-point lacunary polynomial approximation.
\end{abstract}




\section{Introduction}

Pascal's triangle can be represented by the infinite upper triangular matrix

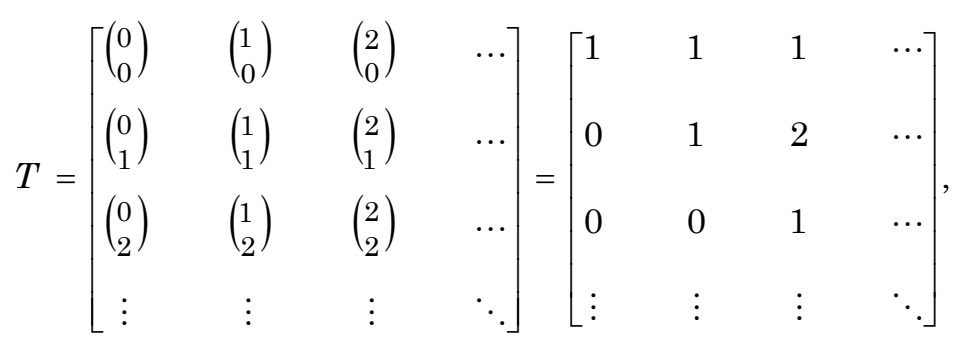

with $\left(\begin{array}{l}j \\ i\end{array}\right):=0$ if $i>j$. Submatrices are of the form

$$
T_{r, c}=\left[\begin{array}{cccc}
\left(\begin{array}{c}
c_{0} \\
r_{0}
\end{array}\right) & \left(\begin{array}{c}
c_{1} \\
r_{0}
\end{array}\right) & \ldots & \left(\begin{array}{c}
c_{n} \\
r_{0}
\end{array}\right) \\
\vdots & \vdots & \ddots & \vdots \\
\left(\begin{array}{c}
c_{0} \\
r_{m}
\end{array}\right) & \left(\begin{array}{l}
c_{1} \\
r_{m}
\end{array}\right) & \cdots & \left(\begin{array}{l}
c_{n} \\
r_{m}
\end{array}\right)
\end{array}\right]
$$

for some selections $r=\left[r_{0}, \ldots, r_{m}\right]$ and $c=\left[c_{0}, \ldots, c_{n}\right]$ of the rows and columns of $T$, respectively. For example,

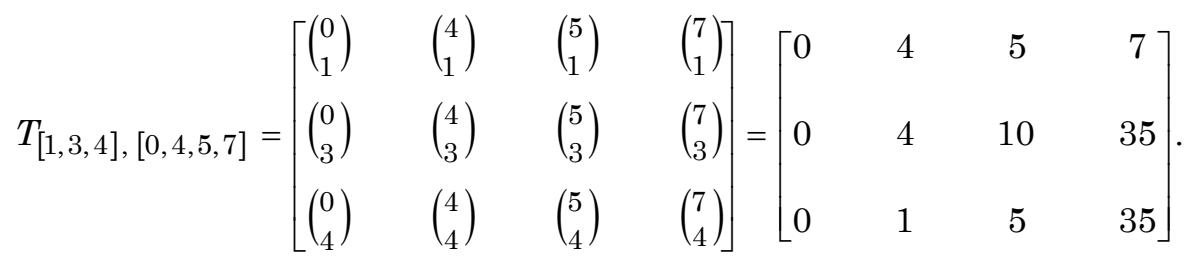

While the rank of this matrix is 3 , it is not obvious to see. The main goal in this paper is to provide a formula for determining the rank of such matrices, and secondly to provide bases for the row and column spaces. Later in the paper, we apply our results to a problem in polynomial approximation. 
The results in this paper are a generalization of the main result in [3], stated below as in Theorem 2.1. This theorem provides necessary and sufficient conditions for the invertibility of square submatrices of the Pascal matrix. In the proof of that result, we showed that the invertibility of square submatrices of $T$ is equivalent to the unique solvability of a two-point Birkhoff interpolation problem. This Birkhoff interpolation problem has been studied in [1, 2, 6, 7], and generalized to lacunary interpolation (see [4, 5]).

In the present paper, we show how to determine the rank of submatrices of the upper triangular Pascal matrix. Our result applies to both square and non-square submatrices. Our main results are stated in Theorem 2.2 and Theorem 3.1. In Algorithm 3.1, we give an algorithm that demonstrates how to compute linearly independent rows and columns. In the final section of this paper, we apply our results to a problem in lacunary polynomial approximation.

\section{Rank of Submatrices of the Pascal Matrix}

In [3], the following result was derived for square submatrices of the Pascal triangle.

Theorem 2.1 ([3, Theorem 1.1]). Let $r=\left[r_{0}, \ldots, r_{m}\right]$ and $c=\left[c_{0}, \ldots, c_{m}\right]$ be indices to the rows and columns of the square submatrix $T_{r, c}$ of the Pascal upper triangular matrix. Then, $T_{r, c}$ is invertible iff the following equivalent conditions hold:

- $r \leq c$ (i.e., $r_{i} \leq c_{i}$ for all $i$ ).

- There is no zero diagonal entry.

Our goal is to generalize this theorem by finding the rank of arbitrary submatrices, square or non-square. These submatrices are defined by sequences of rows $r=\left[r_{0}, \ldots, r_{m}\right]$ and columns $c=\left[c_{0}, \ldots, c_{n}\right]$ of $T$. In general, $m \neq n$. To prove our results, we focus on the condition $r_{i} \leq c_{i}$, and to this end make the following definition: 
Definition 2.1. We say $\{\hat{r}, \hat{c}\}$ is an ordered sub-pair for $\{r, c\}$ of length $p+1$ if $\hat{r}=\left[\hat{r}_{0}, \ldots, \hat{r}_{p}\right]$ is a subsequence of $r=\left[r_{0}, \ldots, r_{m}\right]$, $\hat{c}=\left[\hat{c}_{0}, \ldots, \hat{c}_{p}\right]$ is a subsequence of $c=\left[c_{0}, \ldots, c_{n}\right]$, and $\hat{r} \leq \hat{c}$ (i.e., $\hat{r}_{i} \leq \hat{c}_{i}$ for $\left.i=0, \ldots, p\right)$. If $\hat{r}=\hat{c}=\emptyset$, then $p+1=0$. We say $\{\hat{r}, \hat{c}\}$ is maximal if there is no ordered sup-pair of length greater than $p+1$.

For example, consider $r=[2,7,11,14,17,20]$ and $c=[0,4,9,10,15]$. Then, $\{\hat{r}, \hat{c}\}=\{[2,11],[4,15]\}$ is an ordered sub-pair of length 2 . But this sub-pair is not maximal since the ordered sub-pair $\{[2,7,14],[4,9,15]\}$ is of length 3 , which, as it turns out, is maximal. Note that $\{[2,7,11],[9,10,15]\}$ is also a maximal sub-pair, hence these need not be unique.

The sub-pairs provided in Definition 2.1 are used to construct invertible and full-rank submatrices of $T_{r, c}$, as we summarize in Theorem 2.2.

Theorem 2.2. Let $r=\left[r_{0}, \ldots, r_{m}\right]$ and $c=\left[c_{0}, \ldots, c_{n}\right]$ be indices to the rows and columns of the Pascal upper triangular matrix. Suppose $\{\hat{r}, \hat{c}\}$ is an ordered sup-pair of $\{r, c\}$, with $\hat{r}=\left[\hat{r}_{0}, \ldots, \hat{r}_{p}\right]$ and $\hat{c}=\left[\hat{c}_{0}, \ldots, \hat{c}_{p}\right]$. Then,

- $T_{\hat{r}, \hat{c}}$ is a $(p+1) \times(p+1)$ invertible submatrix of $T_{r, c}$.

- $T_{\hat{r}, c}$ is a $(p+1) \times(n+1)$ submatrix of full row rank.

- $T_{r, \hat{c}}$ is a $(m+1) \times(p+1)$ submatrix of full column rank.

If $\{\hat{r}, \hat{c}\}$ is maximal,

- The rank of $T_{r, c}$ is $p+1$.

- The columns of $T_{r, \hat{c}}$ span the column space of $T_{r, c}$.

- The rows of $T_{\hat{r}, c}$ span the row space of $T_{r, c}$. 
Proof. The dimensions of these submatrices follow from $\# r=m+1$, $\# c=n+1$ and $\# \hat{r}=\# \hat{c}=p+1$. Note that $p \leq \min \{m, n\}$. Since $\{\hat{r}, \hat{c}\}$ is ordered, $\hat{r} \leq \hat{c}$. By Theorem 2.1, $T_{\hat{r}, \hat{c}}$ is invertible. The rank of $T_{\hat{r}, c}$ is at least $p+1$ because it contains the invertible matrix $T_{\hat{r}, \hat{c}}$ as a submatrix. Since it is of dimension $(p+1) \times(n+1)$, it necessarily has a full row rank. Likewise, $T_{r, \hat{c}}$ has full column rank.

For the second part, suppose $\{\hat{r}, \hat{c}\}$ is maximal. Since $T_{\hat{r}, \hat{c}}$ is a submatrix of $T_{r, c}$,

$$
\operatorname{Rank}\left(T_{r, c}\right) \geq \operatorname{Rank}\left(T_{\hat{r}, \hat{c}}\right)=p+1
$$

Suppose $\operatorname{Rank}\left(T_{r, c}\right)>p+1$. Then, there exists rows $\bar{r}=\left[\bar{r}, \ldots, \bar{r}_{p+1}\right]$ and columns $\bar{c}=\left[\bar{c}_{0}, \ldots, \bar{c}_{p+1}\right]$ of $T_{r, c}$ such that $\operatorname{Rank}\left(T_{\bar{r}, \bar{c}}\right)=p+2$. By Theorem 2.1, $\bar{r} \leq \bar{c}$ and so $\{\bar{r}, \bar{c}\}$ is an ordered sup-pair of length $p+2$. But this contradicts the assumption that $\{\hat{r}, \hat{c}\}$ is maximal of order $p+1$. Therefore, $\operatorname{Rank}\left(T_{r, c}\right) \ngtr p+1, \quad$ and so $\operatorname{Rank}\left(T_{r, c}\right)=p+1$,

Finally, if $\{\hat{r}, \hat{c}\}$ is maximal, $\operatorname{Rank}\left(T_{\hat{r}, c}\right)=\operatorname{Rank}\left(T_{r, c}\right)=p+1$, and so $T_{r, c}$. spans the row space of $T_{r, c}$. Likewise, $T_{r, \hat{c}}$ spans the column space of $T_{r, c}$.

\section{Computing the Rank of Submatrices of the Pascal Matrix}

In this section, we show how to compute a maximal ordered sub-pair $\{\hat{r}, \hat{c}\}$ of $\{r, c\}$. In Algorithm 3.1, we actually compute the indices $\alpha$ and $\beta$ of a maximal pair $\{\hat{r}, \hat{c}\}$, such that $\hat{r}=r_{\alpha}=\left[r_{\alpha_{0}}, \ldots, r_{\alpha_{p}}\right]$ and $\hat{c}=c_{\alpha}=\left[c_{\alpha_{0}}, \ldots, c_{\alpha_{p}}\right]$. 
Algorithm 3.1. Compute indices $\{\alpha, \beta\}$ to a maximal ordered subpair of $\{r, c\}$.

- Input $r=\left[r_{0}, \ldots, r_{m}\right]$ and $c=\left[c_{0}, \ldots, c_{n}\right]$.

- If $r_{0}>c_{n}$, set $\alpha=\beta=\emptyset$, go to output.

- Otherwise, set $M:=\max \left\{i: r_{i} \leq c_{n}\right\}$.

- Set $\beta_{0}:=\min \left\{k: r_{0} \leq c_{k}\right\}$.

- For $i=1, \ldots, M$, set $\left.\beta_{i}:=\max \left\{\min \left\{k: r_{i} \leq c_{k}\right\}, \beta_{i-1}+1\right)\right\}$.

- Set $p:=\max \left\{i: \beta_{i} \leq n\right\}$.

- Set $\alpha=\{0, \ldots, p\}$.

- Set $\beta=\left\{\beta_{0}, \ldots, \beta_{p}\right\}$.

- Output $\alpha, \beta$.

The strategy of our algorithm is to pair each $r_{\alpha_{i}}$ with the first $c_{\beta_{i}}$ such that $r_{\alpha_{i}} \leq c_{\beta_{i}}>c_{\beta_{i-1}}$. In this first occurrence strategy, $\alpha=[0, \ldots, p]$. Therefore, $\hat{r}=r_{\alpha}=\left[r_{0}, \ldots, r_{p}\right]$.

Theorem 3.1. Let $r=\left[r_{0}, \ldots, r_{m}\right]$ and $c=\left[c_{0}, \ldots, c_{n}\right]$ be indices to the rows and columns of the rectangular submatrix $T_{r, c}$ of the Pascal upper triangular matrix. Let $\{\alpha, \beta\}$ be determined by Algorithm 3.1. If $\alpha=\beta=\emptyset$, then $T_{r, c}$ is a matrix of zeros of rank 0 . Otherwise, let $\hat{r}=r_{\alpha}$ and $\hat{c}=c_{\beta}$. Then, $\{\hat{r}, \hat{c}\}$ is a maximal ordered sub-pair of $\{r, c\}$, and the rank of the submatrices is determined by Theorem 2.2 .

Proof. Suppose $\alpha=\beta=\emptyset$. By Algorithm 3.1, this occurs when $r_{0}>c_{n}$. In this case, $r_{i}>c_{j}$ for $0 \leq i \leq m$ and $0 \leq j \leq n$, and so the elements $\left(\begin{array}{c}c_{j} \\ r_{i}\end{array}\right)$ of $T_{r, c}$ are all zero. Hence, $T_{r, c}$ is the zero matrix, of rank 0 . 
Suppose that $\alpha$ and $\beta$ are non-empty. This occurs when $r_{0} \leq c_{n}$. Since $\alpha=[0, \ldots, p]$ and $\beta_{i}>\beta_{i-1}$, both $\alpha$ and $\beta$ are strictly increasing sequences. Since $r$ and $c$ are also strictly increasing, it follows that $\hat{r}=r_{\alpha}$ and $\hat{c}=c_{\beta}$ are both strictly increasing. In the algorithm, we choose $\beta_{i}$ to be the first occurrence such that $\alpha_{i} \leq \beta_{i}$ and $\beta_{i}>\beta_{i-1}$. Therefore, the $\beta_{i}$ are incremented by the least amount possible, and so $p$ is the largest index for an ordered sub-pair of $\{r, c\}$. Hence, $\{\hat{r}, \hat{c}\}$ is maximal.

In the next result, we construct index sets of the same rank as $T_{\hat{r}, \hat{c}}$ and $T_{r, c}$ with a minimal number of nonzero entries.

Corollary 3.2. Let $\{\hat{r}, \hat{c}\}$ be an ordered sub-pair of $\{r, c\}$. Let $\alpha$ and $\beta$ be the corresponding index sets such that $\hat{r}=r_{\alpha}$ and $\hat{c}=c_{\beta}$. Let $I_{\hat{r}, \hat{c}}$ be the $(m+1) \times(n+1)$ matrix with $I_{\hat{r}, \hat{c}}\left(\alpha_{i}, \beta_{i}\right)=1$, and all other entries zero. Then, $\operatorname{Rank}\left(T_{\hat{r}, \hat{c}}\right)=\operatorname{Rank}\left(I_{\hat{r}, \hat{c}}\right)$. If $\{\hat{r}, \hat{c}\}$ is maximal, then $\operatorname{Rank}\left(T_{r, c}\right)=\operatorname{Rank}\left(I_{\hat{r}, \hat{c}}\right)$.

Proof. By Theorem 2.2, the rank of $T_{\hat{r}, \hat{c}}$ is $p+1$. This is the number of elements in $\hat{r}$ and $\hat{c}$, which equals the number of elements in $\alpha$ and $\beta$. The matrix $I_{\hat{r}, \hat{c}}$ has zeros for all entries except for entry $\left(\alpha_{i}, \beta_{i}\right)$ for $i=0, \ldots, p$. But, since $\alpha$ and $\beta$ are strictly increasing, these nonzero entries are in different rows and columns. Hence, the dimension of the row and column spaces of $I_{\hat{r}, \hat{c}}$ are necessarily equal to the number of nonzero elements, which is the length of $\alpha$ or $\beta$. Therefore, the rank of $I_{\hat{r}, \hat{c}}$ is $p+1$, which equals the rank of $T_{\hat{r}, \hat{c}}$.

In the case that $\{\hat{r}, \hat{c}\}$ is maximal, we have by Theorem 2.2 that the rank of $T_{r, c}$ equals the rank of $T_{\hat{r}, \hat{c}}$, which equals the rank of $I_{\hat{r}, \hat{c}}$.

We conclude this section with a constructive example. 
Example 3.2. Let $r=\left[r_{0}, \ldots, r_{5}\right]=[2,7,11,14,17,20]$ and $c=\left[c_{0}, \ldots, c_{4}\right]=[0,4,9,10,15]$. These are the rows and columns for the following submatrix of the Pascal matrix:

$$
T_{r, c}=T_{[2,7,11,14,17,20],[0,4,9,10,15]}=\left[\begin{array}{ccccc}
0 & 6 & 36 & 45 & 105 \\
0 & 0 & 36 & 120 & 6435 \\
0 & 0 & 0 & 0 & 1365 \\
0 & 0 & 0 & 0 & 15 \\
0 & 0 & 0 & 0 & 0 \\
0 & 0 & 0 & 0 & 0
\end{array}\right] .
$$

Then, $m=5$ and $n=4$, and

$$
M=\max _{i}\left\{r_{i} \leq c_{4}\right\}=\max _{i}\left\{r_{i} \leq 15\right\}=3,
$$

since $14 \leq 15$ but $17 \nless 15$. Then,

$\beta_{0}=\left\{\min _{k}\left(r_{0} \leq c_{k}\right)\right\}=\left\{\min _{k}\left(2 \leq c_{k}\right)\right\}=1$ since $c_{0}<2 \leq c_{1}$,

$\beta_{1}=\max \left\{\min _{k}\left(r_{1} \leq c_{k}\right), \beta_{0}+1\right\}=\max \left\{\min _{k}\left(7 \leq c_{k}\right), 1+1\right\}=\max \{2,2\}=2$,

$\beta_{2}=\max \left\{\min _{k}\left(r_{2} \leq c_{k}\right), \beta_{1}+1\right\}=\max \left\{\min _{k}\left(11 \leq c_{k}\right), 2+1\right\}=\max \{4,3\}=4$,

$\beta_{3}=\max \left\{\min _{k}\left(r_{3} \leq c_{k}\right), \beta_{2}+1\right\}=\max \left\{\min _{k}\left(14 \leq c_{k}\right), 4+1\right\}=\max \{4,5\}=5$,

and so

$$
\beta=\left[\beta_{i}: \beta_{i} \leq n\right]=\left[\beta_{i}: \beta_{i} \leq 4\right]=\left[\beta_{0}, \beta_{1}, \beta_{2}\right]=[1,2,4] .
$$

Hence, $p=2$. Then, $\hat{r}=\eta_{[0,1,2]}=[2,7,11]$ and $\hat{c}=c_{[1,2,4]}=[4,9,15]$.

Thus, we compute

$$
T_{\hat{r}, \hat{c}}=T_{[2,7,11],[4,9,15]}=\left[\begin{array}{ccc}
6 & 36 & 105 \\
0 & 36 & 6435 \\
0 & 0 & 1365
\end{array}\right] .
$$


By Theorem 2.2,

$$
\operatorname{Rank}\left(T_{r, c}\right)=\operatorname{Rank}\left(T_{\hat{r}, \hat{c}}\right)=p+1=3,
$$

and $T_{\hat{r}, \hat{c}}$ is invertible. Also by this theorem, the matrix

$$
T_{\hat{r}, c}=T_{\left[r_{0}, r_{1}, r_{2}\right], c}=T_{[2,7,11],[0,4,9,10,15]}
$$

has linearly independent rows (full row rank), and the matrix

$$
T_{r, \hat{c}}=T_{r,\left[c_{1}, c_{2}, c_{4}\right]}=T_{[2,7,11,14,17,20],[4,9,15]}
$$

has linear independent columns (full column rank). The index matrix $I_{\hat{r}, \hat{c}}$ defined in Corollary 3.2 is nonzero only at entries $\left(\alpha_{i}, \beta_{i}\right)$. These are $(0,1),(1,2)$, and $(2,4)$. Hence,

$$
I_{\hat{r}, \hat{c}}=\left[\begin{array}{ccccc}
0 & 1 & 0 & 0 & 0 \\
0 & 0 & 1 & 0 & 0 \\
0 & 0 & 0 & 0 & 1 \\
0 & 0 & 0 & 0 & 0 \\
0 & 0 & 0 & 0 & 0 \\
0 & 0 & 0 & 0 & 0
\end{array}\right],
$$

which is rank 3.

\section{Application to Polynomial Approximation}

In [3], we established a connection between submatrices of the Pascal upper triangular matrix and polynomial interpolation. Let $r=\left[r_{0}, \ldots, r_{m}\right]$ and $c=\left[c_{0}, \ldots, c_{n}\right]$. Let

$$
\Lambda_{x, r}=\left[\frac{\delta_{x} D^{r_{0}}}{r_{0} !}, \ldots, \frac{\delta_{x} D^{r_{m}}}{r_{m} !}\right]
$$

with

$$
\delta_{x} D^{r_{i}}: f \mapsto f^{\left(r_{i}\right)}(x),
$$


and let

$$
V_{c}=\left[(\cdot)^{c_{0}}, \ldots,(\cdot)^{c_{n}}\right]
$$

be the power basis with powers $c_{i}$ for $i=0, \ldots, n$. Then, the matrix

$$
\Lambda_{x, r}^{T} V_{c}=\left[\frac{\delta_{x} D^{r_{i}}(\cdot)^{c_{j}}}{c_{j} !}\right]
$$

is a kind of generalized Vandermonde for the one-point lacunary polynomial interpolation problem. This kind of matrix has been considered in [4, 5], but for square matrices. In [3], we showed that this generalized Vandermonde, for the case that $x=1$, is related to the Pascal matrix as follows:

Proposition 4.1. Let $r=\left[r_{0}, \ldots, r_{m}\right]$ and $c=\left[c_{0}, \ldots, c_{n}\right]$ be selections of the rows and columns of the Pascal upper triangular matrix T. Then, $T_{r, c}=\Lambda_{1, r}^{T} V_{c}$.

Hence, by Theorem 3.1, we have the following:

Corollary 4.2. Let $r=\left[r_{0}, \ldots, r_{m}\right]$ and $c=\left[c_{0}, \ldots, c_{n}\right]$. Assume $r_{0} \leq c_{n}$. Let $\{\hat{r}, \hat{c}\}$ be an ordered sub-pair of $\{r, c\}$. Then, $\Lambda_{1, r}^{T} V_{\hat{c}}$ has full column rank.

Now we will apply our result to polynomial approximation. Let $\{\hat{r}, \hat{c}\}$ be an ordered sub-pair of $\{r, c\}$ of length $p+1$. We may assume it is maximal, but it doesn't need to be. Let

$$
f(x)=\sum_{j=0}^{p} b_{j} x^{\hat{c}_{j}} .
$$

Assume we are given some data, $y=\left[y_{0}, \ldots, y_{m}\right]$. Since typically $m>p$, we cannot expect to interpolate. However, by Corollary 4.2, $\Lambda_{1, r}^{T} V_{\hat{c}}$ has full column rank, and so we can easily solve the least squares problem 


$$
\operatorname{Minimize}\left\{\sum_{i=0}^{m}\left\|D^{r_{i}} f(1)-y_{i}\right\|_{2}^{2}, b \in \mathbb{R}^{p+1}\right\} .
$$

The unique solution to this problem is

$$
b=A^{+} y=\left(A^{T} A\right)^{-1} A^{T} y,
$$

where $A^{+}$is the pseudo-inverse of $A:=\Lambda_{1, r}^{T} V_{\hat{c}}$.

We conclude with an example.

Example 4.1. Let $r=\left[r_{0}, \ldots, r_{5}\right]=[2,7,11,14,17,20]$ and $c=\left[c_{0}, \ldots, c_{4}\right]=[0,4,9,10,15]$. In Example 3.2, it was shown that $\{\hat{r}, \hat{c}\}=\{[2,7,11],[4,9,15]\}$ is a maximal ordered sub-pair of $\{r, c\}$. Therefore, $\hat{c}=[4,9,15]$ is the degree sequence of our lacunary polynomial, and

$$
A=\Lambda_{1, r}^{T} V_{\hat{c}}=\left[\begin{array}{ccc}
6 & 36 & 105 \\
0 & 36 & 6435 \\
0 & 0 & 1365 \\
0 & 0 & 15 \\
0 & 0 & 0 \\
0 & 0 & 0
\end{array}\right] \text {. }
$$

Suppose $y=[1,1,1,1,1,1]^{T}$. Then, the coefficient sequence of the least squares solution is

$$
b=\left[\begin{array}{lll}
.7813 & -.1046 & .0007
\end{array}\right]^{T} .
$$

Hence, the least squares lacunary polynomial is

$$
f(x)=.7813 x^{4}-.1046 x^{9}+.0007 x^{15} .
$$




\section{References}

[1] G. D. Birkhoff, General mean value and remainder theorems with applications to mechanical differentiation and integration, Trans. Amer. Math. Soc. 1 (1906), 107-136.

[2] D. Ferguson, The question of uniqueness for G. D. Birkhoff interpolation problems, J. Approx. Th. 2 (1969), 1-28.

[3] S. Kersey, Invertibility of submatrices of the Pascal matrix and Birkhoff interpolation, Journal of Mathematical Sciences: Advances and Applications 41 (2016), 45-56.

[4] F. Palacios-Quiñonero, P. Rubió-Díaz, J. Díaz-Barrero and J. Rossell, Order regularity of two-node Birkhoff interpolation with lacunary polynomials, Applied Mathematics Letters 22 (2009), 386-389.

[5] F. Palacios-Quiñonero, P. Rubió-Díaz, J. Díaz-Barrero and J. Rossell, Order of regularity for Birkhoff interpolation with lacunary polynomials, Mathematica Aeterna 1(3) (2011), 129-135.

[6] G. Pölya, Bemerkungen zur Interpolation und zur Näherungstheorie der Balkenbiegung, Z. Angew. Math. Mech. 11 (1931), 4445-4449.

[7] J. M. Whittaker, Interpolatory Function Theory, Cambridge University Press, London, 1935. 